\title{
Resistance to Noise of Non-linear Observers in Canonical Form Application to a Sludge Activation Model
}

\author{
Benoît Schwaller ${ }^{1}$, Denis Ensminger ${ }^{1}$, Birgitta Dresp-Langley ${ }^{2} \&$ José Ragot $^{3}$ \\ ${ }^{1}$ Laboratory of Inventive Design (LGECO), EA 3938 INSA Strasbourg, Université de Strasbourg, France \\ ${ }^{2}$ Laboratory of Engineering, Informatics and Imaging (ICUBE), Université de Strasbourg, UMR 7357 CNRS, France \\ ${ }^{3}$ Centre de Recherche en Automatique de Nancy (CRAN), Université de Lorraine, UMR 7039 CNRS, France
}

Correspondence: B. Schwaller, IUT Louis Pasteur de Strasbourg, département Génie Industriel et Maintenance, 1 Allée d'Athènes, 67300 Schiltigheim. E-mail: schwaller@ convergence.u-strasbg.fr

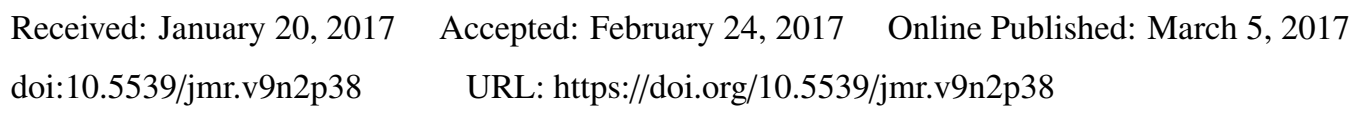

\begin{abstract}
The aim of this study was to increase the resistance to noise of an observer of a non-linear MISO system transformed into canonical regulation form of order $n$. For this, the principle idea was to add $n$ observers on the output equations of the main observer. By adjusting the time scale of the output observers, the resistance to noise of the final estimates is considerably increased. The proposed method is illustrated by model simulations based on a non-linear Sludge Activation Model (SAM)
\end{abstract}

Keywords: non-linear systems, state observers, continuous time

\section{Introduction}

State observers have been intensely exploited since (Luenberger, 1966), to model, control or identify linear and non-linear systems, including the studies of (Krener \& Isidori, 1983; Zheng, Boutat, \& Barbot, 2009) relating to non-linear systems transformable into a canonical form. The key idea in such approaches is to produce approximate measures of non-linearity of order 1, as in Extended Luenberger Observers (ELO) (Ciccarella, Mora, \& Germani, 1993). Approximations of nonlinearities in the canonical form (which results in ELO) have already been suggested (Bestle \& Zeitz, 1983), and this approach can be extended to higher order approximations (Röbenack \& Lynch, 2004). An observer using a partial nonlinear observer canonical form (POCF) (Röbenack \& Lynch, 2006) has weaker observability and integrability existence conditions than the well-established non-linear observer canonical form (OCF). Non-linear sliding mode observers use a quasi-Newtonian approach, applied after pseudo-derivations of the output signal (Efimov \& Fridman, 2011). State observers using Extended Kalman Filters (EKF) provide another method of transforming non-linear systems (Boker \& Khalil, 2013), (Rauh, Butt, \& Aschemann, 2013). Finding an appropriate method for parameter synthesis remains one of the major difficulties with state observers for non-linear systems. (Tornambè, 1992), (Mobki, Sadeghia, \& Rezazadehb, 2015) proposed high-gain state observers to deal with this problem. High-gain state observers reduce observation errors for a range of predetermined amplitudes or fluctuations by making the observations independent of parameters. The weak point of this method is its sensitivity to noise and uncertainty.

In network identification and encryption, observers with delays are used to synchronize chaotic oscillators, as shown in several studies (Ibrir, 2009; Martínez-Guerra, et al., 2011). Noise and uncertainty are not critical factors in such a context. This can be very different in the case of industrial processes, as shown in a recent study (Bodizs, 2011), where the performances of observers using ELO, EKF or Integrated Kalman Filters (IKF) are compared. The influence of noise and uncertainty on these observer types was emphasized, with more reliable results produced by ELO observers, which permit the exact state reconstruction of highly perturbed systems. For PI and ELO observer classes, (Söffker, et al., 2002) demonstrated a compensation effect on measurement errors ; (Khalifa \& Mabrouk, 2015) addressed the problem of uncertainty of non-linear models. One way of overcoming the problem of parametric uncertainty is to use adaptive observers (Tyukina, et al., 2013; Farza, et al., 2014) in the particular case where the measurements are only available at discrete instants and have disturbances. Another approach (Mazenc \& Dinh, 2014; Thabet, et al., 2014) consists of defining interval observers. Modeling observer systems by Takagi-Sugeno decomposition (Bezzaoucha, et al., 2013; Guerra, et al., 2015) is another possibility, as is the use of models using symmetries and semi-invariants (Menini \& Tornambè, 2011), or the use of immersible techniques for systems transformed into a non-linear observer form (Back \& Seo, 2008).

A large number of non-linear MISO systems with multiple inputs and a single output can be transformed into state 
equations using the form :

$$
\begin{aligned}
\underline{\dot{z}}(t) & =\underline{s}(t) \\
y(t) & =\underline{d}^{T} \underline{z}(t)+\Phi(t) \\
\underline{s}(t) & =\left[\begin{array}{c}
s_{1}\left[\underline{z}(t), \underline{u}_{1}(t)\right] \\
\ldots \\
s_{n}\left[\underline{z}(t), \underline{u}_{1}(t)\right]
\end{array}\right] \\
\underline{d}^{T} & =\left[\begin{array}{lll}
d_{1} & \ldots & d_{n}
\end{array}\right]
\end{aligned}
$$

with the following definitions :

$n:$ the order of the system of non-linear differential equations

$m:$ number of independant inputs

$\underline{u}_{1}(t)^{T}:$ the vector $\left[u_{11}(t), \ldots u_{m 1}(t)\right]$ of the $m$ independent inputs

$y(t)$ : the measurable output variable

$\underline{z}^{T}(t):$ the state vector $\left[z_{1}(t) \ldots z_{n}(t)\right]$

$\underline{d}^{T}:$ the vector of the output parameters of the system

$\bar{\Phi}(t)$ : the non-linear function of vector $\underline{u}_{1}(t)$ of the inputs

$s_{i}\left[\underline{z}(t), \underline{u}_{1}(t)\right]:$ one of the $n$ non-linear functions of the state vector $\underline{s}(t)$.

Such systems are often found in nonlinear robotic systems in the form of trigonometric functions. Other systems contain non-linear polynomials (strange attractors, Bernouilli functions, non-linear springs), polynomial fractions, or various common simple functions...

The $n$ non-linear functions of vector $\underline{s}(t)$ employ a vector of $m$ independent inputs $\underline{u}_{1}(t)$, as well as the state vector $\underline{z}(t)$ as input variables. Such a procedure allows amongst other possibilities the description of bi-linear systems. We limited ourselves in this study to continuous functions in all points of type $C^{1}$.

One considers that the measurable output is a linear combination of $z(t)$, superimposed on a non-linear function $\Phi\left[\underline{u}_{1}(t)\right]$. For an engineer or physicist, many applications have such a form. Often, non-linear systems (1) are transformable in a regulation canonical form concieved by (Fliess, 1990), and are written :

$$
\begin{aligned}
\underline{\dot{x}}(t) & =\underline{A} \underline{x}(t)+\underline{f}(t) \\
y(t) & =\underline{c}^{T} \underline{x}(t)+\Phi(t) \\
\underline{A} & =\delta_{i j} \quad j=i+1, \quad i=1 \ldots n-1 \\
\underline{c}^{T} & =\left[\begin{array}{lll}
c_{1} & \ldots & c_{n}
\end{array}\right] \\
\underline{f}(t)^{T} & =\left[\begin{array}{llll}
0 & \ldots & 0 & \Psi[\underline{x}(t), \underline{U}(t)
\end{array}\right]
\end{aligned}
$$

with the following definitions :

$\underline{u}_{i}(t)$ : the $(i-1)-t h$ temporal derivative of the vector $\underline{u}_{1}(t)$, either $\underline{u}_{i}(t)^{T}=\left[u_{1 i}(t), \ldots u_{m i}(t)\right] \quad i=2 \ldots n$ $\underline{U}(t)=\left[\underline{u}_{1}(t) \ldots \underline{u}_{n}(t)\right]:$ the $n \times m$ input matrix, with the group of $n$ vectors $\underline{u}_{i}(t)$

$x_{i}(t):(i-1)$ th temporal derivative of $x_{1}(t)$

$\underline{x}^{T}(t):$ state vector $\left[x_{1}(t), \ldots x_{n}(t)\right]$

$\underline{\underline{c}}$ : the output parameters vector of the transformed system

$\bar{\theta} \leqslant n:$ index of last coefficient $c_{i} \neq 0$

$\Psi[\underline{x}(t), \underline{U}(t)]:$ a scalar non-linear $C^{1}$ function

$\underline{A}$ : the $n \times n$ matrix of which the last line is zero.

Conversion of the transformed version (2) to the initial representation (1) is performed using :

$$
\begin{aligned}
\underline{z}(t) & =\underline{g}(t) \\
\underline{g}(t)^{T} & =\left[g_{1}[\underline{x}(t), \underline{U}(t)] \ldots g_{n}[\underline{x}(t), \underline{U}(t)]\right]
\end{aligned}
$$

with $\underline{g}(t)$ : the vector of $n$ non-linear inverted transformation functions $g_{i}[\underline{x}(t), \underline{U}(t)]$ which link $\underline{x}(t)$ to $\underline{z}(t)$.

In (Schwaller, Ensminger, Dresp-Langley, \& Ragot, 2016) a new observer was proposed which was adapted to this transformed form, and which provided non-biased robust estimates of $\underline{x}(t)$. This is not always the case for estimates of $\underline{z}(t)$. Functions $g_{i}[\underline{x}(t)](1 \mathrm{~b})$ permit linking $\underline{x}(t)$ to $\underline{z}(t)(2 \mathrm{c})$ and are called inverted transformations. Because of the nonlinearity of $\underline{g}(t)$, small perturbations of estimates of $\underline{x}(t)$ may be considerably increased and strongly disturb estimates 
of $\underline{z}(t)$. The main aim of this study was to solve this type of situation, by introducing the inverted observer transform functions $g_{i}[\underline{x}(t)]$. Doing this, the resistance to observer noise is affected (Bodizs et al., 2011), and one obtains a tool capable of limiting its impact on estimates of $\underline{z}(t)$.

Definition 1 Let us define, for the moment, a normalised pulse $\omega_{o}=2 \pi / T_{o}$, which introduces a new time scale $\tau$ for the representation of the transformed state of the system:

$$
\begin{aligned}
\underline{\dot{x}}(\tau) & =\underline{A} \underline{x}(\tau)+\underline{f}(\tau) \\
y(\tau) & =\underline{\widetilde{c}}^{T} \underline{x}(\tau)+\Phi(\tau) \\
\tilde{\underline{c}}^{T} & =\left[\begin{array}{lll}
\widetilde{c}_{1} & \ldots & \widetilde{c}_{n}
\end{array}\right] \\
\underline{f}(\tau)^{T} & =\left[\begin{array}{llll}
0 & \ldots & 0 & \widetilde{\Psi}[\underline{x}(\tau), \underline{U}(\tau)]
\end{array}\right]
\end{aligned}
$$

and for the inverse transformation system :

$$
\begin{aligned}
\underline{z}(\tau) & =\underline{g}(\tau) \\
\underline{g}(\tau)^{T} & =\left[\begin{array}{llll}
g_{1}[\underline{x}(\tau), \underline{U}(\tau)] & \ldots & g_{n}[\underline{x}(\tau), \underline{U}(\tau)]
\end{array}\right]
\end{aligned}
$$

with :

$$
\begin{aligned}
\tau & =\omega_{o} t, \quad \dot{x}_{n}(t)=\dot{x}_{n}(\tau) \omega_{o}{ }^{n} \\
u_{i j}(t) & =u_{i j}(\tau) \omega_{o}{ }^{i-1}, \quad x_{i}(t)=x_{i}(\tau) \omega_{o}{ }^{i-1} \\
\widetilde{c}_{i} & =c_{i} \omega_{o}{ }^{i-1}, \quad z_{i}(t)=z_{i}(\tau), \quad i=1 \ldots n
\end{aligned}
$$

$\underline{f}(\tau)$ and $\underline{g}(\tau)$ are vectors with dimension $n$. In $(4 \mathrm{~b}), \Phi(\tau)=\Phi\left[\underline{u}_{1}(\tau)\right]=\Phi\left[\underline{u}_{1}(t)\right]$. Equations (6) define time dilatation or retraction of the state representation and its new parameters, without changing the pattern of the signal $x_{i}(\tau)$. For the function $\Psi$, this is translated by the relation of changing the following scale representation :

$$
\Psi[\underline{x}(t), \underline{U}(t)]=\omega_{o}{ }^{n} \widetilde{\Psi}[\underline{x}(\tau), \underline{U}(\tau)]
$$

The function $\widetilde{\Psi}[\underline{x}(\tau), \underline{U}(\tau)]$ is obtained by replacing every state or command variable by the corresponding one in (6) and dividing everything by $\omega_{o}{ }^{n}$.

Afterwards, the procedure can be separated into several steps: in section 2, the estimation of the state of the transformed system (4) is dealt with ; in section 3 a new observation method of the inverse transformation functions which permit estimation of state variables (1) is presented ; in section 4 this new approach is applied to observe a system of management of activated sludge in a purification station; the study is concluded in section 5 .

\section{Structure of the Observer in Canonical Form}

To begin with, let us isolate the componant $x_{1}(\tau)$ of (4b) which will subsequently serve to determine the observation error. To obtain $y_{1}(\tau)$, the estimation of variable $x_{1}(\tau)$, three cases may be distinguished. For $\theta=1$ :

$$
y_{1}(\tau)=\frac{y(\tau)-\Phi(\tau)}{\widetilde{c}_{1}}
$$

For $\theta=2$, it becomes :

$$
\dot{y}_{1}(\tau)=-\frac{\widetilde{c}_{1}}{\widetilde{c}_{2}} y_{1}(\tau)+\frac{y(\tau)-\Phi(\tau)}{\widetilde{c}_{2}}
$$

In the most general case where $\theta>2, y(\tau)-\Phi(\tau)$ is filtered by :

$$
\begin{aligned}
\underline{\dot{w}}(\tau) & =\underline{K} \underline{w}(\tau)+\underline{k}[y(\tau)-\Phi(\tau)] \\
\underline{K} & =\left[\begin{array}{cccc}
0 & 1 & 0 & \ldots \\
0 & 0 & 1 & \ldots \\
\ldots & 0 & 0 & 1 \\
-\widetilde{c}_{1} & \ldots & \ldots & -\frac{\tilde{c}_{\theta-1}}{\widetilde{c}_{\theta}}
\end{array}\right] \\
\underline{w}(\tau)^{T} & =\left[\begin{array}{llll}
y_{1}(\tau) & \ldots & y_{\theta-1}(\tau)
\end{array}\right], \quad \underline{w}(0)=\underline{0} \\
\underline{k}^{T} & =\left[\begin{array}{llll}
0 & \ldots & 0 & 1 / \widetilde{c}_{\theta}
\end{array}\right]
\end{aligned}
$$


To analyze the effect of the filter, we rewrite (4b) in scalar form, ignoring $\widetilde{c}_{\theta+1} \ldots \widetilde{c}_{n}$, which are all zero :

$$
y(\tau)-\Phi(\tau)=\sum_{i=1}^{\theta} \widetilde{c}_{i} x_{i}(\tau)
$$

If (11) is inserted in (10a), (9) or (8) as a function of $\theta$, it becomes :

$$
\begin{aligned}
\sum_{i=1}^{\theta} \widetilde{c}_{i} y_{i}(\tau) & =\sum_{i=1}^{\theta} \widetilde{c}_{i} x_{i}(\tau) \\
\dot{y}_{\theta-1}(\tau) & =y_{\theta}(\tau) \quad \theta \geqslant 2
\end{aligned}
$$

The Laplace transformation of (12a) gives the transfer function :

$$
y_{1}(s) / x_{1}(s)=1
$$

To develop the rest, $y_{1}(\tau)$ is used to determine the observer error.

Definition 2 To generate state estimates $\underline{v}(\tau)$ for the system (4), a PI observer structure is defined in (Schwaller, Ensminger, Dresp-Langley, $\mathcal{E}$ Ragot, 2016) with :

$$
\begin{aligned}
& \underline{\dot{\tilde{x}}}(\tau)=\underline{A} \underline{\breve{x}}(\tau)+\underline{\tilde{f}}(\tau)+\underline{\breve{h}} \Delta y_{1}(\tau) \\
& \underline{\dot{\hat{x}}}(\tau)=\underline{A} \underline{\hat{x}}(\tau)+\underline{\check{A}} \underline{\breve{x}}(\tau)+\underline{\hat{h}} \Delta y_{1}(\tau) \\
& \Delta y_{1}(\tau)=x_{1}(\tau)-\widehat{x}_{1}(\tau) \\
& \underline{\tilde{f}}(\tau)^{T}=\left[\begin{array}{llll}
0 & \ldots & 0 & \tilde{f}(\tau)
\end{array}\right] \\
& \dot{I}_{0}(\tau)=h_{0} \Delta y_{1}(\tau) \\
& \tilde{f}(\tau)=I_{0}(\tau)+\widetilde{\Psi}[\underline{v}(\tau), \underline{U}(\tau)] \\
& \underline{\check{x}}(\tau)^{T}=\left[\begin{array}{lll}
\breve{x}_{2}(\tau) & \ldots & \breve{x}_{n}(\tau)
\end{array}\right] \\
& \underline{\hat{x}}(\tau)^{T}=\left[\begin{array}{lll}
\hat{x}_{1}(\tau) & \ldots & \hat{x}_{n-1}(\tau)
\end{array}\right] \\
& \underline{v}(\tau)^{T}=\left[\begin{array}{ll}
\hat{x}_{1}(\tau) & \underline{\check{x}}(\tau)^{T}
\end{array}\right] \\
& \underline{\hat{x}}(0)=\underline{\breve{x}}(0)=\underline{0}, \quad I_{0}(0)=0 \\
& \underline{\underline{h}}^{T}=\left[\begin{array}{ll}
\underline{0}^{T} & h_{1}
\end{array}\right] \\
& \underline{\hat{h}}^{T}=\left[\begin{array}{lll}
h_{n} & \ldots & h_{2}
\end{array}\right] \\
& \underline{h}^{T}=\left[\underline{\hat{h}}^{T}, \quad h_{1}\right] \\
& \underline{A}=\delta_{i j}, \quad j=i+1, i=1 \ldots n-1 \\
& \underline{A}=\left[\begin{array}{cccc}
0 & \ldots & \ldots & 0 \\
\vdots & \ddots & \ldots & \vdots \\
\vdots & & 0 & \vdots \\
0 & \ldots & \ldots & 1
\end{array}\right]
\end{aligned}
$$

with $\underline{\check{x}}(\tau)(14 g)$ and $\underline{\hat{x}}(\tau)(14 h)$ as two distinct state vectors of dimension $n-1$, coupled using the matrices $\underline{A}(14 n)$ and $\underline{\breve{A}}$ $(140)$ of dimension $(n-1) \times(n-1)$. The vectors $\underline{\underline{h}}$ and $\underline{\underline{h}}$ are also of dimension $n-1$. The matrix $\underline{A}$ is constructed using the Kronecker operator which puts the upper diagonal at 1 . The parameters $h_{i}, i=0 \ldots n$ are the gains of the observer.

Figure 1 illustrates the functional diagram of such an observer of third order.

The augmented vector $\underline{v}(\tau)(14 \mathrm{i}),(14 \mathrm{~h})$ and $(14 \mathrm{~g})$ is used as estimation of $\underline{x}(\tau)$ and as variable of the function $\tilde{\Psi}[\underline{v}(\tau), \underline{U}(\tau)]$ (14f). The state $\underline{\hat{x}}(\tau)(14 \mathrm{~b})$ is an observer exploiting the observation error $\Delta y_{1}(\tau)(14 \mathrm{c})$ via the gains $h_{i}(14 \mathrm{~m})$ serving to correct the state distances between the system and its observer. 


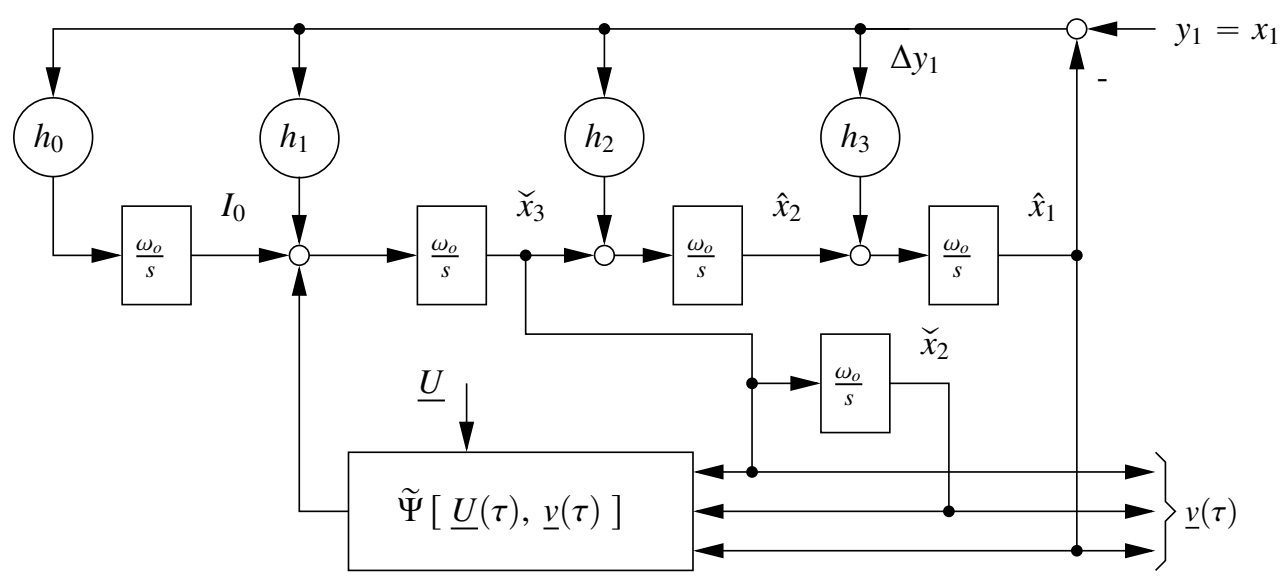

Figure 1. Third order observer

In figure 1, for example, we have :

$$
\begin{aligned}
& \underline{\hat{x}}(\tau)^{T}=\left[\begin{array}{ll}
\widehat{x}_{1}(\tau) & \hat{x}_{2}(\tau)
\end{array}\right] \\
& \underline{\check{x}}(\tau)^{T}=\left[\begin{array}{ll}
\breve{x}_{2}(\tau) & \breve{x}_{3}(\tau)
\end{array}\right] \\
& \underline{v}(\tau)^{T}=\left[\begin{array}{lll}
\hat{x}_{1}(\tau) & \check{x}_{2}(\tau) & \check{x}_{3}(\tau)
\end{array}\right] \\
& \underline{\hat{h}}^{T}=\left[\begin{array}{ll}
h_{3} & h_{2}
\end{array}\right] \\
& \stackrel{\breve{h}}{ }^{T}=\left[\begin{array}{ll}
0 & h_{1}
\end{array}\right]
\end{aligned}
$$

The choice of using two state variables $\underline{\hat{x}}(\tau)$ and $\underline{\breve{x}}(\tau)$ is motivated by the $n-1$ successive integrations of $\dot{\breve{x}}_{n}(\tau)$ in which no $\underline{\hat{h}} \Delta y_{1}(\tau)$ re-injection error is involved. This allows an increase in the robustness of the estimations to the measurement noise, which in general affects the variable $y_{1}(\tau)$. One thus overcomes a common weak point of high gain observations, i.e. their sensitivity to measurement noise. The second advantage comes from the non-linear function $\widetilde{\Psi}[\underline{v}(\tau), \underline{U}(\tau)]$ which is no longer subjected to the restrictive conditions used in (Schwaller, Ensminger, Dresp-Langley, \& Ragot, 2013), and covers the ensemble of the systems described by (Fliess, 1990). The vector $\widetilde{f}(\tau)(14 \mathrm{~d})$, of dimension $n-1$, compensates the effects of $f(\tau)$, and of possible external exogenous disturbance of (2) using the integral component $I_{0}(\tau)(14 \mathrm{e})$. One notes that at the second order, for a gain $h_{0}=0$ inhibiting the integrator $I_{0}$, the observer becomes similar to that proposed by (Gauthier, Hammouri, \& Othman, 1992) for a SISO system.

In (Schwaller, Ensminger, Dresp-Langley, \& Ragot, 2016), a full analysis was performed in order to determine the dynamics of the observation error $\Delta y_{1}(\tau)(14 \mathrm{c})$ and its successive derivatives, to characterise stability conditions and also the exponential convergent nature of estimates $\underline{v}(\tau)$. A mthod to synthesize parameters $h_{0} \ldots h_{n}$ was also proposed.

\section{Observation of the Original System via the Inverted Transformation Functions}

\subsection{New observers definitions}

In (5b), the inverted transform functions $g(\tau)$ allow converting the system in the canonical form of regulation back to the original form (1). Using the estimates $\underline{v}(\bar{\tau})$ reconstructed by the observer (14), it is possible to define : (15)

$$
\begin{aligned}
\widehat{z}_{i}(t) & =\widehat{g}_{i}[\underline{v}(\tau), \underline{U}(\tau)] \quad i=1 \ldots n \\
\underline{\hat{z}}(t)^{T} & =\left[\begin{array}{lll}
\widehat{z}_{1}(t) & \ldots & \widehat{z}_{n}(t)
\end{array}\right]
\end{aligned}
$$

One thus obtains estimates $\underline{\underline{z}}(t)$ of $\underline{z}(t)$ (1). If the stability conditions (Theorem 1 of (Schwaller, Ensminger, Dresp-Langley, $\&$ Ragot, 2016)) are respected, $\underline{\hat{z}}(t) \rightarrow \underline{z}(t)$ when $\Delta y_{1}(t) \rightarrow 0$. Similarly, $\underline{\hat{z}}(t) \rightarrow \underline{\dot{z}}(t)$ when $\Delta y_{1}(t) \rightarrow 0$. One then has :

$$
\begin{aligned}
\lim _{\Delta y_{1}(t) \rightarrow 0^{-}} \underset{\hat{z}}{\underline{z}}(t) & =\underline{\dot{z}}(t) \\
\underline{\hat{z}}(t)^{T} & =\left[\begin{array}{lll}
\widehat{s}_{1}(t) & \ldots & \widehat{s}_{n}(t)
\end{array}\right] \\
\widehat{s}_{i}(t) & =s_{i}\left[\underline{\underline{z}}(t), \underline{u}_{1}(t)\right] \quad i=1 \ldots n
\end{aligned}
$$




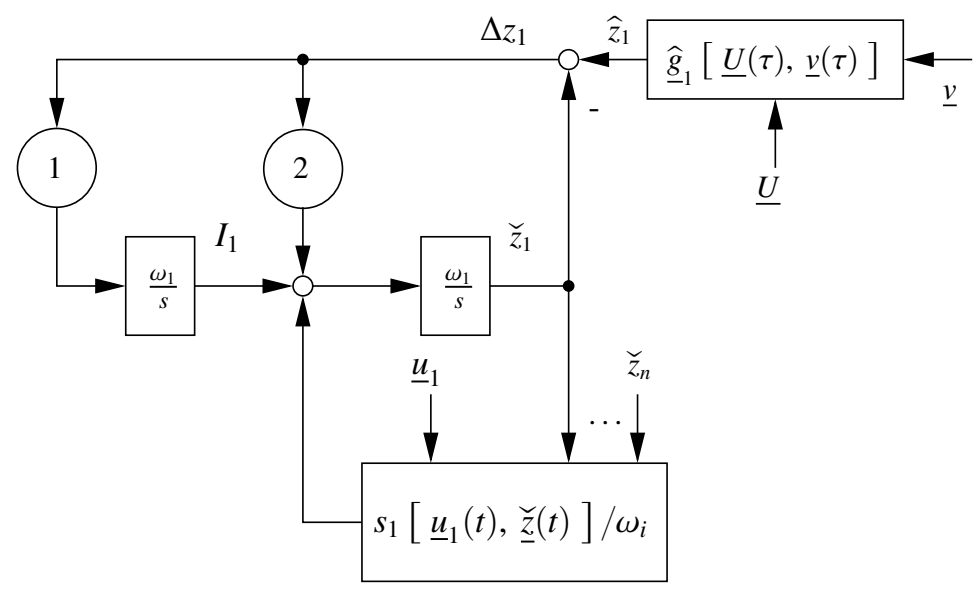

Figure 2. Observer of inverse function $\widehat{z}_{1}\left(\tau_{1}\right)$

The $n$ estimates $\widehat{z}_{i}(t)$ can be used as reference inputs to observe $n$ state variables $\breve{z}_{i}(t)$ which tend towards (15a). Their temporal derivatives tend towards $\underline{\hat{z}}(t)$, which themselves tend towards $\dot{z}(t)$ (16). With the model (14), one defines $n$ first order observers. Each is normalised by a pulse $\omega_{i}$ which leads to its dimensionless time definition (17e), possesses its own Lipschitz constant, and its specific stability conditions that we have to find. Synthesising the gains $h_{i}$ (subsection 2.4 of (Schwaller, Ensminger, Dresp-Langley, \& Ragot, 2016)) gives $h_{0}=1$ and $h_{1}=2$. The $n$ observers are written :

$$
\begin{aligned}
\dot{\check{z}}_{i}\left(\tau_{i}\right) & =I_{i}\left(\tau_{i}\right)+2 \Delta z_{i}\left(\tau_{i}\right)+\check{s}_{i}\left(\tau_{i}\right) \\
\dot{I}_{i}\left(\tau_{i}\right) & =\Delta z_{i}\left(\tau_{i}\right) \quad i=1 \ldots n \\
\Delta z_{i}\left(\tau_{i}\right) & =\widehat{z}_{i}\left(\tau_{i}\right)-\check{z}_{i}\left(\tau_{i}\right) \\
\check{s}_{i}\left(\tau_{i}\right) & =s_{i}\left[\check{z}(t), \underline{u}_{1}(t)\right] / \omega_{i} \\
\tau_{i} & =\omega_{i} t
\end{aligned}
$$

with $\underline{z}(t)=\left[\begin{array}{lll}\breve{z}_{1}(t) & \ldots & \breve{z}_{n}(t)\end{array}\right]$ the vector of the estimations of $\underline{z}(t) ; \check{s}_{i}\left(\tau_{i}\right)$ is the normalised non-linear function of $\dot{\check{z}}_{i}\left(\tau_{i}\right)$. Figure 2 illustrates (15) and (17).

The general calculation procedure is as follows :

- estimation of $\underline{v}(\tau)(14 \mathrm{i})$ after treatment of (14);

- estimation of $\underline{\underline{z}}(t)(15)$;

- estimation of the $n$ state distances (17c);

- determination of the $n$ non-linear functions $\breve{s}_{i}\left(\tau_{i}\right)(17 \mathrm{~d})$ to access the $n$ terms $\dot{\check{z}}_{i}\left(\tau_{i}\right)(17 \mathrm{a})$ and $\dot{I}_{i}\left(\tau_{i}\right)(17 \mathrm{~b})$;

- integration of the $n$ equations (17a) to obtain $\underline{z}(t)$.

The temporal derivative of (17c) and inserting (17a) in the rest obtained enables one to obtain the expression of $\Delta \dot{z}_{i}(\tau)$ :

$$
\begin{aligned}
\Delta \dot{z}_{i}\left(\tau_{i}\right) & =\dot{\widehat{z}}_{i}\left(\tau_{i}\right)-\dot{\check{z}}_{i}\left(\tau_{i}\right) \quad i=1 \ldots n \\
& =\Delta \widetilde{\Psi}_{i}\left(\tau_{i}\right)-I_{i}\left(\tau_{i}\right)-2 \Delta z_{i}\left(\tau_{i}\right) \\
\Delta \widetilde{\Psi}_{i}\left(\tau_{i}\right) & =\widehat{s}_{i}\left(\tau_{i}\right)-\breve{s}_{i}\left(\tau_{i}\right) \\
\widehat{s}_{i}\left(\tau_{i}\right) & =s_{i}\left[\widehat{z}(t), \underline{u}_{1}(t)\right] / \omega_{i}
\end{aligned}
$$

\subsection{Dynamics of the Observer Errors}

We now characterise the dynamics of the observer errors by searching the $n$ differential equations of the distances $\Delta z_{i}\left(\tau_{i}\right)$. Due to the presence of integrators $I_{i}\left(\tau_{i}\right)$, an extra temporal derivative is necessary to obtain the differential equation of the 
distances $\Delta z_{i}\left(\tau_{i}\right)$. To do this, it is necessary to define the following augmented vectors :

$$
\begin{aligned}
\underline{\Upsilon}\left(\tau_{i}\right) & =\left[\begin{array}{ll}
\underline{u}_{1}\left(\tau_{i}\right) & \underline{u}_{2}\left(\tau_{i}\right)
\end{array}\right] \\
\underline{\hat{z}}_{i}\left(\tau_{i}\right)^{T} & =\left[\begin{array}{ll}
\widehat{z}_{i}\left(\tau_{i}\right) & \dot{\hat{z}}_{i}\left(\tau_{i}\right)
\end{array}\right] \quad i=1 \ldots n \\
\check{z}_{i}\left(\tau_{i}\right)^{T} & =\left[\begin{array}{ll}
\check{z}_{i}\left(\tau_{i}\right) & \dot{\check{z}}_{i}\left(\tau_{i}\right)
\end{array}\right] \\
\Delta \underline{z}_{i}\left(\tau_{i}\right)^{T} & =\left[\begin{array}{lll}
\Delta z_{i}\left(\tau_{i}\right) & \Delta \dot{z}_{i}\left(\tau_{i}\right)
\end{array}\right] \\
\underline{\hat{Z}}\left(\tau_{i}\right)^{T} & =\left[\begin{array}{lll}
\underline{\underline{z}}_{1}\left(\tau_{i}\right) & \ldots & \widehat{\underline{z}}_{n}\left(\tau_{i}\right)
\end{array}\right] \\
\underline{\check{Z}}\left(\tau_{i}\right)^{T} & =\left[\begin{array}{lll}
\check{\underline{z}}_{1}\left(\tau_{i}\right) & \ldots & \check{z}_{n}\left(\tau_{i}\right)
\end{array}\right] \\
\Delta \underline{Z}\left(\tau_{i}\right)^{T} & =\left[\begin{array}{lll}
\Delta \underline{z}_{1}\left(\tau_{i}\right) & \ldots & \Delta \underline{z}_{n}\left(\tau_{i}\right)
\end{array}\right]
\end{aligned}
$$

The temporal derivative of $(18 \mathrm{~b})$ is written :

$$
\begin{aligned}
\Delta \ddot{z}_{i}\left(\tau_{i}\right) & =\Delta \dot{\tilde{\Psi}}_{i}\left(\tau_{i}\right)-\Delta z_{i}\left(\tau_{i}\right)-2 \Delta \dot{z}_{i}\left(\tau_{i}\right) \quad i=i \ldots n \\
\Delta \dot{\tilde{\Psi}}_{i}\left(\tau_{i}\right) & =\dot{s}_{i}\left[\underline{\widehat{Z}}\left(\tau_{i}\right), \underline{\Upsilon}\left(\tau_{i}\right)\right]-\dot{s}_{i}\left[\underline{\check{Z}}\left(\tau_{i}\right), \underline{\Upsilon}\left(\tau_{i}\right)\right]
\end{aligned}
$$

and gives the scalar expression of the differential equations of the observation errors. Using notations (19) gives the matricial writing of (20a) in the form of state equations :

$$
\begin{aligned}
\Delta \dot{z}_{i}\left(\tau_{i}\right) & =\underline{A}_{i} \Delta \underline{z}_{i}\left(\tau_{i}\right)+\Delta \underline{\tilde{\Psi}}_{i}\left(\tau_{i}\right) \quad i=1 \ldots n \\
\underline{A}_{i} & =\left[\begin{array}{cc}
0 & 1 \\
-1 & -2
\end{array}\right] \\
\Delta \underline{\tilde{\Psi}}_{i}\left(\tau_{i}\right) & =\left[\begin{array}{c}
0 \\
\Delta \dot{\widetilde{\Psi}}_{i}\left(\tau_{i}\right)
\end{array}\right]
\end{aligned}
$$

Assuming that the non-linear functions $\dot{s}_{i}$ are at least locally Lipschitz in $\underline{Z}\left(\tau_{i}\right)$, and uniformly bounded in $\underline{\Upsilon}\left(\tau_{i}\right)$ in an invariant set, they are associated with a Lipschitz constant $L_{i}$ :

$$
\left\|\Delta \dot{\widetilde{\Psi}}_{i}\left(\tau_{i}\right)\right\| \leqslant L_{i}\left\|\Delta \underline{Z}\left(\tau_{i}\right)\right\| \quad i=1 \ldots n
$$

Applying the Lipschitz inequality to (20b) permits reduction to $\Delta \underline{Z}\left(\tau_{i}\right)$ the number of useful variables to characterise the perturbing difference $\Delta \dot{\widetilde{\Psi}}_{i}\left(\tau_{i}\right)$. For many systems, if functions $\dot{s}_{i}$ are not globally of a Lipschitz type, they can be locally or be transformed adequately into the Lipschitz type.

\subsection{Convergence of State Observations}

Now let us try to analyse the globally asymptotic development of the observation errors and to characterise the limiting stability conditions of each observer (17).

Theorem 1 Let us consider a MISO system decomposable as described in (4), for which the observer structures (14) and (17) are used, and related to each other by the inverted transform function (15a). If the system function $\dot{s}_{i}\left[\underline{\hat{Z}}\left(\tau_{i}\right), \underline{\Upsilon}\left(\tau_{i}\right)\right]$ is locally of the Lipschitz type in $\underline{\underline{Z}}\left(\tau_{i}\right)$ and uniformly bounded in $\underline{\Upsilon}\left(\tau_{i}\right)$ in an invariant set, with a Lipschitz constant $L_{i}$ (22), then the observer (17) will be locally stable if the Lipschitz constant $L_{i}$ satisfies the following conditions :

$$
\begin{aligned}
& L_{i}^{2} \leqslant \frac{2 \sigma_{i} \phi_{i 1}-1}{4 n \sigma_{i}^{2} \phi_{i 1}\left(\frac{\lambda_{i}{ }^{2}}{\phi_{i 1}}+\frac{\phi_{i 1}}{4}\right)} \\
& L_{i}{ }^{2} \leqslant \frac{2 \sigma_{i}\left(4 \phi_{i 2}-\phi_{i 1}\right)-1}{4 n \sigma_{i}^{2} \phi_{i 2}\left(\phi_{i 2}+\frac{\phi_{i 1}{ }^{2}}{4 \phi_{i 2}}\right)} \\
& \lambda_{i}>0, \quad \sigma_{i}>0, \quad \phi_{i 1}>0, \quad \phi_{i 2}>0 \quad i=0 \ldots n
\end{aligned}
$$


If the system function $\dot{s}_{i}\left[\underline{\widehat{Z}}\left(\tau_{i}\right), \underline{\Upsilon}\left(\tau_{i}\right)\right]$ is globally of the Lipschitz type, and if the Lipschitz constant $L_{i}$ satisfy (23), then the observers (17) will be globally asymptotically stable.

Proof. The proof of theorem 1 can be demonstrated by proving the stability of (21a) using an appropriate positive Lyapunov function, like the following quadratic function :

$$
\begin{aligned}
\breve{V}_{n}\left(\tau_{i}\right) & =\sum_{i=1}^{n} v_{i}\left(\tau_{i}\right) \\
v_{i}\left(\tau_{i}\right) & =\Delta \underline{z}_{i}\left(\tau_{i}\right)^{T} \underline{P}_{i} \Delta \underline{z}_{i}\left(\tau_{i}\right) \\
\underline{P}_{i} & =\left[\begin{array}{cc}
\lambda_{i} & 0 \\
\phi_{i 1} & \phi_{i 2}
\end{array}\right]
\end{aligned}
$$

The $\underline{P}_{i}$ lower triangular matrix are defined as positive and satisfying the Sylvester criteria, with (24c). The proof of convergence is linked to the study of the sign of the derivative of the candidate for a Lyapunov function. This is obtained after temporal derivation of (24a), and after placing (21a) in the result obtained for terms $\Delta \dot{z}_{i}\left(\tau_{i}\right)$ :

$$
\begin{aligned}
& \dot{\grave{V}}_{n}\left(\tau_{i}\right)=\sum_{i=1}^{n} \dot{v}_{i}\left(\tau_{i}\right) \\
& \dot{v}_{i}\left(\tau_{i}\right)=\Delta \underline{z}_{i}\left(\tau_{i}\right)^{T} \underline{Q}_{i} \Delta \underline{z}_{i}\left(\tau_{i}\right)+N_{i}\left(\tau_{i}\right) \quad i=0 \ldots n \\
& \underline{Q}_{i}=\underline{A}_{i}^{T} \underline{P}_{i}+\underline{P}_{i} \underline{A}_{i} \\
& =\left[\begin{array}{cc}
-\phi_{i 1} & 0 \\
2\left(\lambda_{i}-\phi_{i 1}\right)-\phi_{i 2} & \phi_{i 1}-4 \phi_{i 2}
\end{array}\right] \\
& N_{i}\left(\tau_{i}\right)=\Delta \underline{z}_{i}\left(\tau_{i}\right)^{T} \underline{S}_{i} \Delta \underline{\tilde{\Psi}}_{i}\left(\tau_{i}\right) \\
& \underline{S}_{i}=\underline{P}_{i}+\underline{P}_{i}^{T}
\end{aligned}
$$

An appropriate choice of $\phi_{i 1}, \phi_{i 2}$ can provide negative diagonal coefficients for $\underline{Q}_{i}$. The criterion of semi-negativity of Sylvester is then respected, and the successive minors of $\underline{Q}_{i}$ will be of opposite sign, ensuring the semi-negativity of the first member on the right of (25b). Verifying the sign of the second member on the right of (25b) involves increasing $N_{i}\left(\tau_{i}\right)$ using the inequalities of Schwartz and Lipschitz (22) :

$$
\begin{aligned}
N_{i}\left(\tau_{i}\right) & \leqslant\left\|\Delta \underline{z}_{i}\left(\tau_{i}\right)^{T} \underline{S}_{i} \Delta \underline{\dot{\tilde{\Psi}}}\left(\tau_{i}\right)\right\| \\
& \leqslant\left\|\Delta \underline{z}_{i}\left(\tau_{i}\right)^{T} \underline{S}_{i}\right\|\left\|\Delta \underline{\tilde{\Psi}}\left(\tau_{i}\right)\right\| \\
& \leqslant\left\|\Delta \underline{z}_{i}\left(\tau_{i}\right)^{T} \underline{S}_{i}\right\| L\left\|\Delta \underline{z}_{i}\left(\tau_{i}\right)\right\|
\end{aligned}
$$

To determine the sign of $\dot{v}_{i}\left(\tau_{i}\right)$ function, one applies the following inequality :

$$
\begin{aligned}
\left\|\underline{a}\left(\tau_{i}\right)^{T} \underline{b}\left(\tau_{i}\right)\right\| & \leqslant \frac{n \sigma_{i}}{2} \underline{a}\left(\tau_{i}\right)^{T} \underline{a}\left(\tau_{i}\right)+\frac{1}{2 n \sigma_{i}} \underline{b}\left(\tau_{i}\right)^{T} \underline{b}\left(\tau_{i}\right) \\
\underline{a}\left(\tau_{i}\right) & =L \underline{S}_{i}^{T} \underline{z}_{i}\left(\tau_{i}\right) \\
\underline{b}\left(\tau_{i}\right) & =\Delta \underline{z}_{i}\left(\tau_{i}\right)
\end{aligned}
$$

to (26c) to obtain the desired increase of $N_{i}\left(\tau_{i}\right)$ :

$$
\begin{aligned}
N_{i}\left(\tau_{i}\right) & \leqslant \Delta \underline{z}_{i}\left(\tau_{i}\right)^{T} \underline{R}_{i} \Delta_{z_{i}}\left(\tau_{i}\right) \quad i=1 \ldots n \\
\underline{R}_{i} & =\frac{n \sigma_{i} L_{i}^{2}}{2} \underline{S}_{i} \underline{S}_{i}+\frac{\underline{I}}{2 n \sigma_{i}}
\end{aligned}
$$

In (28a) yields a positive lower triangular matrix $\underline{R}_{i}(28 \mathrm{~b})$, the diagonal elements of which are written :

$$
r_{j j}=\left\{\begin{array}{cc}
2 n \sigma_{i} L_{i}{ }^{2}\left(\lambda_{i}{ }^{2}+\phi_{i 1}{ }^{2} / 4\right)+\frac{1}{2 \sigma_{i}} & j=1 \\
2 n \sigma_{i} L_{i}{ }^{2}\left(\phi_{i 2}{ }^{2}+\phi_{i 1}{ }^{2} / 4\right)+\frac{1}{2 \sigma_{i}} & j=2
\end{array}\right.
$$


The inequality (28a) permits deduction of (25b) :

$$
\begin{aligned}
\dot{v}_{i}\left(\tau_{i}\right) & \leqslant \Delta \underline{z}_{i}\left(\tau_{i}\right)^{T} \underline{M}_{i} \Delta \underline{z}_{i}\left(\tau_{i}\right) \\
\underline{M}_{i} & =\underline{Q}_{i}+\underline{R}_{i}
\end{aligned}
$$

With negative functions $\dot{v}_{i}\left(\tau_{i}\right)$, adding together the diagonal terms of (25c) and (29), and imposing $Q_{i}+\underline{R}_{i} \leqslant 0$, one obtains the conditions (23). The sum $\underline{Q}_{i}+\underline{R}_{i}$ yields an inferior triangular matrix that satisfies Sylvester criteria of seminegativity if inequalities (23a) and (23b) are satisfied. Then, if $\Delta \dot{\widetilde{\Psi}}_{i}\left(\tau_{i}\right)(20 \mathrm{~b})$ is Lipschitz $(22), \dot{v}_{i}\left(\tau_{i}\right)$ is semi-negative and (21a) is globally and asymptotically stable ; (21a) is locally stable if (22) is locally Lipschitz

Using the (theorem 2, section 2.3, (Schwaller, Ensminger, Dresp-Langley, \& Ragot, 2016)), it is easy to demonstrate that the observers (17) will be exponentially convergent.

\section{Application to a Sludge Activation Model}

Let us now illustrate the proposed observation method by applying it to a non-linear example with multiple inputs.

\subsection{Original Model}

For this we choose a simplified treatment model for activated sludge ASM1 similar to that used by (Nagy-Kiss et al., 2010), and structurally of the same types as (1):

$$
\begin{aligned}
\dot{z}_{i}(t) & =s_{i}(t) \quad i=1 \ldots 3 \\
y_{1}(t) & =z_{1}(t) \quad y_{2}(t)=z_{2}(t)
\end{aligned}
$$

with :

$$
\begin{aligned}
& s_{1}(t)=k_{1} \ell_{1}(t)+k_{2} z_{3}(t)-k_{3} \ell_{2}(t) z_{3}(t) \\
& s_{2}(t)=k_{4} \ell_{3}(t)-k_{1} \ell_{4}(t)-k_{5} \ell_{2}(t) z_{3}(t) \\
& s_{3}(t)=k_{9} \ell_{5}(t)-k_{6} \ell_{6}(t)-k_{7} z_{3}(t)+k_{8} \ell_{2}(t) z_{3}(t)
\end{aligned}
$$

and non-linear functions :

$$
\begin{aligned}
\ell_{1}(t) & =u_{11}(t)\left(u_{31}(t)-z_{1}(t)\right) \\
\ell_{2}(t) & =\frac{z_{1}(t) z_{2}(t)}{\left(k_{10}+z_{1}(t)\right)\left(k_{11}+z_{2}(t)\right)} \\
\ell_{3}(t) & =u_{21}(t)\left[k_{12}-z_{2}(t)\right] \\
\ell_{4}(t) & =u_{11}(t) z_{2}(t) \\
\ell_{5}(t) & =u_{11}(t) u_{41}(t) \\
\ell_{6}(t) & =u_{11}(t) z_{3}(t) \\
\underline{u}_{1}(t) & =\left[\begin{array}{lll}
u_{11}(t) & \ldots & u_{41}(t)
\end{array}\right]
\end{aligned}
$$

The constants used are given by :

$$
\begin{array}{lll}
k_{1}=5 \cdot 10^{-11} & k_{2}=1,08 \cdot 10^{-5} & k_{3}=2.872 \cdot 10^{-4} \\
k_{4}=3.510^{-4} & k_{5}=9 \cdot 10^{-5} & k_{6}=1.316 \cdot 10^{-12} \\
k_{7}=4.8 \cdot 10^{-6} & k_{8}=7.47 \cdot 10^{-5} & k_{9}=8 \cdot 10^{-11} \\
k_{10}=20 & k_{11}=0.2 & k_{12}=10
\end{array}
$$

$\underline{u}_{1}(t)(33 \mathrm{~g})$ represent the inputs of the system (figures 3(a),(b),(c),(d) page 10), respectively the input flow of waste water, the flow of injected air, the concentration soluble carbonated substrate recycled, the particle concentration of recycled heterotrophic biomass. All abscissas of the figures are expressed in hours.

The variables $z_{1}(t), z_{2}(t) z_{3}(t)$ represent the state of the reactor (figures 3(e),(f), (g)), respectively the concentration of rapidly biodegradable substrate, the concentration of dissolved oxygen, the particle concentration of biomass, with (34) its parameters, all known, and $z_{1}(0)=4.1, z_{2}(0)=3.0, z_{3}(0)=867$ the initial conditions. The sizes $y_{1}(t), y_{2}(t)(31 \mathrm{~b})$ represent the measurable outputs. 


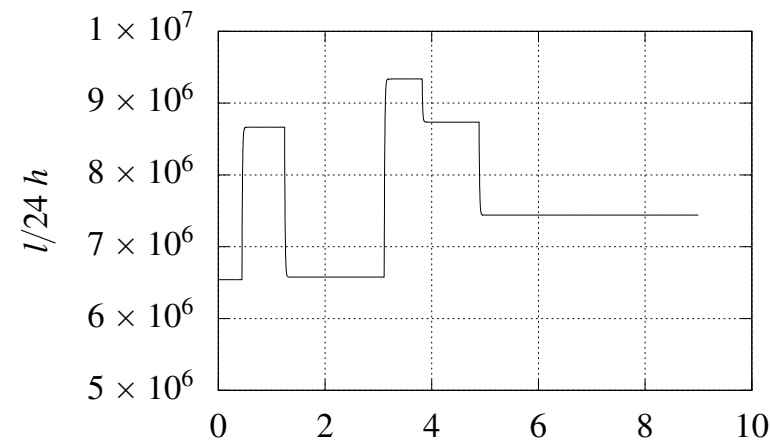

(a) $u_{11}(t)$, water flow

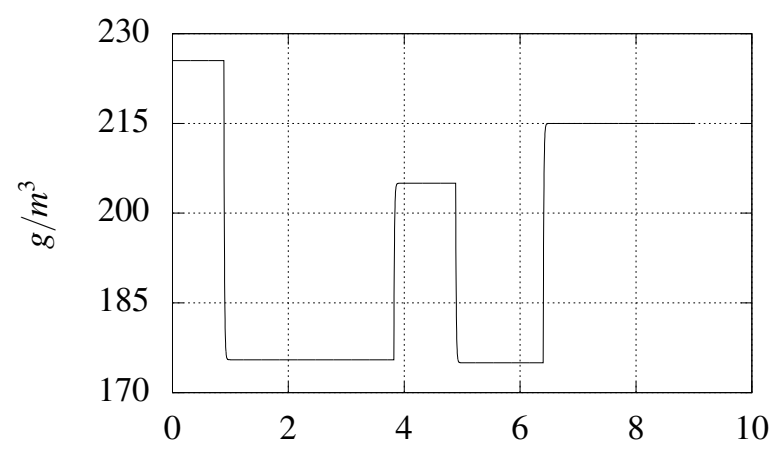

(c) $u_{31}(t)$, injection of carbonated substrate

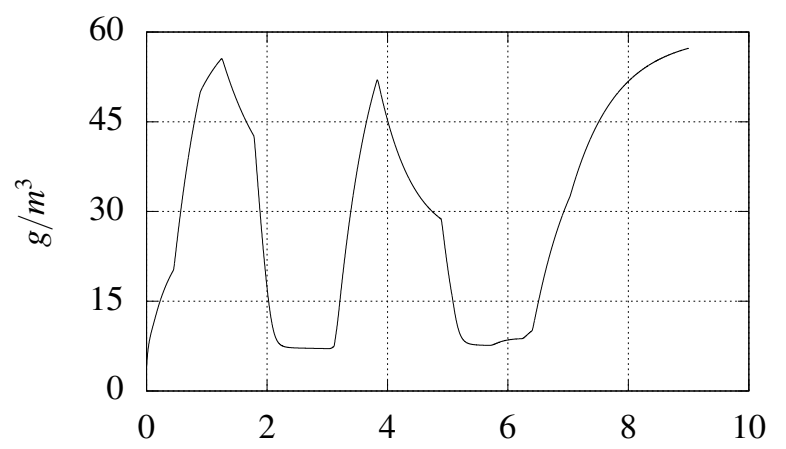

(e) $z_{1}(t)$, concentration of biodegradable carbonated substrate

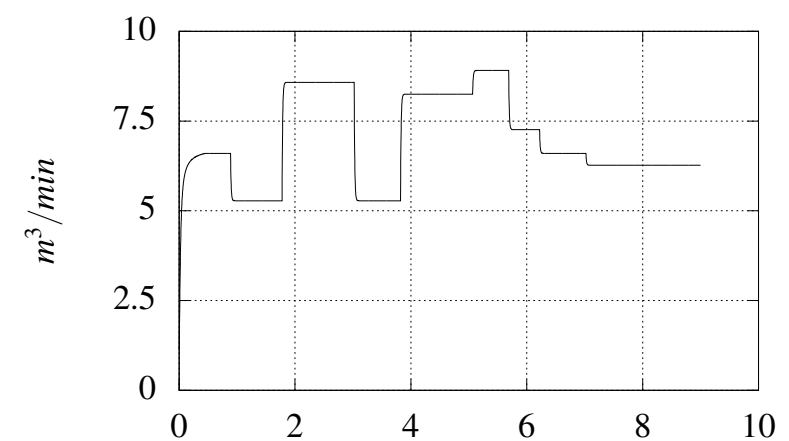

(b) $u_{21}(t)$, air flow

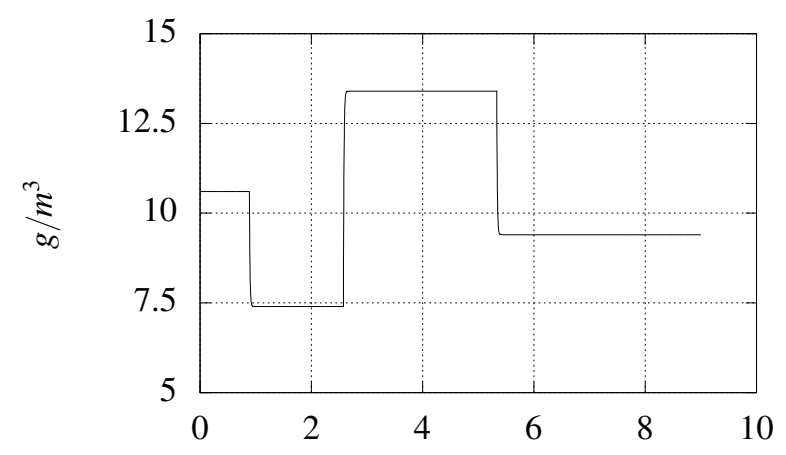

(d) $u_{41}(t)$, injection of biomass

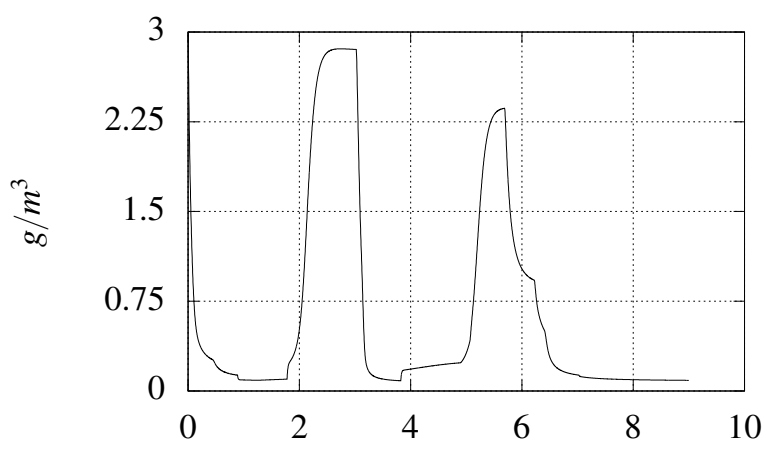

(f) $z_{2}(t)$, concentration of dissolved oxygen

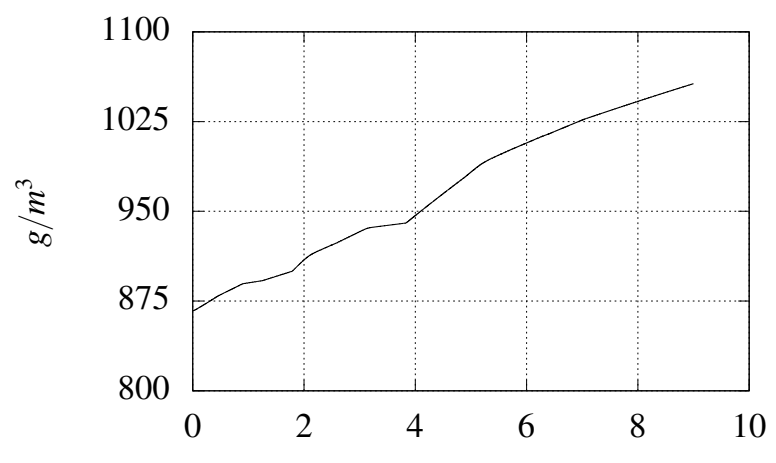

(g) $z_{3}(t)$, concentration of biomass

Figure 3. Input variables and state variables of the bioreactor 


\subsection{Model Transformed in a Canonical Form of Regulation}

We now transform the two first order differential equations (32b) and (32c) into a single second order differential equation. With this one we determine a function $\Psi[\underline{x}(t), \underline{U}(t)]$ and a new differential equation in a canonical form of regulation. As this application of the general procedure of transformation permits passage from systems (1) to (2) (Fliess, 1990) it will permit the use of an observer similar to that proposed in (14), associated with inverted transformation (15) and with observers (17).

In our example we try to observe the measured output $y_{2}(t)$ and its successive derivatives, to subsequently determine an estimation of the immeasurable variable $z_{3}(t)$. From $s_{2}(t)(32 \mathrm{~b})$ we deduce $z_{3}(t)$ :

$$
z_{3}(t)=\frac{k_{4} \ell_{3}(t)-k_{1} \ell_{4}(t)-\dot{z}_{2}(t)}{k_{5} \ell_{2}(t)}
$$

The temporal derivative of (35) gives an expression of $\dot{z}_{3}(t)$ which can be equated to $s_{3}(t)$ (32c). We thus deduce :

$$
\begin{aligned}
\dot{x}_{1}(t) & =x_{2}(t) \\
\dot{x}_{2}(t) & =\Psi[\underline{x}(t), \underline{U}(t)] \\
\Psi[\underline{x}(t), \underline{U}(t)] & =k_{4} \dot{\ell}_{3}(t)-k_{1} \dot{\ell}_{4}(t)-k_{5} \ell_{7}(t) \\
\ell_{2}(t) & =\frac{z_{1}(t) x_{1}(t)}{\left(k_{10}+z_{1}(t)\right)\left(k_{11}+x_{1}(t)\right)} \\
& =n(t) / d(t) \\
\ell_{3}(t) & =u_{21}(t)\left[k_{12}-x_{1}(t)\right] \\
\ell_{4}(t) & =u_{11}(t) x_{1}(t) \\
\ell_{6}(t) & =u_{11}(t) z_{3}(t) \\
\ell_{7}(t) & =z_{3}(t) \dot{\ell}_{2}(t)+s_{3}(t) \ell_{2}(t) \\
z_{2}(t) & =x_{1}(t)
\end{aligned}
$$

System (36) is made up of a second order differential equation, in a canonical regulation form structurally of the same type as that described in (2). The derived functions $\dot{\ell}_{3}(t), \dot{\ell}_{4}(t)$ of $(36 \mathrm{c})$, and $\dot{\ell}_{2}(t), s_{3}(t)$ of $(36 \mathrm{~h})$ are defined by :

$$
\begin{aligned}
\dot{\ell}_{2}(t) & =\dot{n}(t) d(t)-\dot{d}(t) n(t) / d(t)^{2} \\
\dot{n}(t) & =s_{1}(t) x_{1}(t)+z_{1}(t) x_{2}(t) \\
\dot{d}(t) & =s_{1}(t)\left(k_{11}+x_{1}(t)\right)+x_{2}(t)\left(k_{10}+z_{1}(t)\right) \\
s_{1}(t) & =k_{1} \ell_{1}(t)+k_{2} g_{3}(t)-k_{3} \ell_{2}(t) g_{3}(t) \\
\dot{\ell}_{3}(t) & =u_{22}(t)\left(k_{12}-x_{1}(t)\right)-u_{21}(t) x_{2}(t) \\
\dot{\ell}_{4}(t) & =u_{12}(t) x_{1}(t)+u_{11}(t) x_{2}(t) \\
s_{3}(t) & =k_{9} \ell_{5}(t)-k_{6} \ell_{6}(t)-k_{7} g_{3}(t)+k_{8} \ell_{2}(t) g_{3}(t)
\end{aligned}
$$

Equation $\dot{z}_{1}(t)=s_{1}(t)$ defined in (31a) is conserved, and the integration of $s_{1}(t)$ provides $z_{1}(t)$, which is the measured output variable defined in (31b). The system of equation of functions of inverted transforms (3b) should permit in our example determination of $z_{3}(t)$. It is written :

$$
\begin{aligned}
& z_{1}(t)=g_{1}(t) \\
& z_{2}(t)=g_{2}(t) \\
& z_{3}(t)=g_{3}(t)=\frac{k_{4} \ell_{3}(t)-k_{1} \ell_{4}(t)-x_{2}(t)}{k_{5} \ell_{2}(t)}
\end{aligned}
$$

and permits linking $\underline{x}(t)$ to $\underline{z}(t): z_{3}(t)$ is a non-linear function of $\underline{U}(t)$ and $\underline{x}(t)$ through $\ell_{2}(t), \ell_{3}(t)$ and $\ell_{4}(t)$. 
4.3 Time Scaling of $\tilde{\Psi}[\underline{U}(t), \underline{x}(t)]$, Observation of $z_{2}(t)$ in Canonical Form of Regulation and Determination of the Inverted Transform System

Using (7), one can temporally normalise (36b), putting for $\widetilde{\Psi}(\tau)$ the definition of the following input-output variables :

$$
\begin{aligned}
& \widetilde{\Psi}(\tau)=\widetilde{\Psi}\left[\underline{v}_{a}(t), \underline{U}(t)\right] / \omega_{o}^{2} \\
& \underline{v}_{a}(t)^{T}=\left[\begin{array}{ll}
\breve{z}_{1}(t) & \underline{v}(t)
\end{array}\right] \\
& \underline{v}(t)^{T}=\left[\begin{array}{ll}
\widehat{x}_{1}(t) & \check{x}_{2}(t)
\end{array}\right] \\
& \breve{x}_{2}(t)=\omega_{o} \breve{x}_{2}(\tau) \quad \widehat{x}_{1}(t)=\widehat{x}_{1}(\tau) \\
& \underline{U}(t)=\left[\underline{u}_{1}(t) \quad \underline{u}_{2}(t)\right]
\end{aligned}
$$

The scaling pulse chosen for (39) is $\omega_{o}=3.92710^{-2} \mathrm{rd} / \mathrm{s}$. In (39b) we define $\underline{v}_{a}(t)$ as the vector $\underline{v}(t)(14 \mathrm{i})$ augmented by variable $\breve{z}_{1}(t)$, itself resulting from observation of the measured variable $y_{1}(t)$.

Note that $\underline{v}(t)$ contains two second order terms because of (36). Equation (39d) allows conversion of time scaled state variables to temporal variables. Taking (7) and definitions (39) into account, function $\widetilde{\Psi}(\tau)$ in scaled time used in (39a) is written :

$$
\begin{aligned}
\widetilde{\Psi}(\tau) & =\left(k_{4} \dot{\hat{\ell}}_{3}(t)-k_{1} \dot{\hat{\ell}}_{4}(t)-k_{5} \hat{\ell}_{7}(t)\right) / \omega_{o}^{2} \\
\hat{\ell}_{1}(t) & =u_{11}(t)\left(u_{31}(t)-\check{z}_{1}(t)\right) \\
\hat{\ell}_{2}(t) & =\widehat{n}(t) / \hat{d}(t) \\
\hat{\ell}_{3}(t) & =u_{21}(t)\left[k_{12}-\widehat{x}_{1}(t)\right] \\
\hat{\ell}_{4}(t) & =u_{11}(t) \hat{x}_{1}(t) \\
\hat{\ell}_{5}(t) & =u_{11}(t) u_{41}(t) \\
\hat{\ell}_{6}(t) & =u_{11}(t) \hat{z}_{3}(t) \\
\hat{\ell}_{7}(t) & =\widehat{z}_{3}(t) \hat{\hat{\ell}}_{2}(t)+\dot{\hat{z}}_{3}(t) \hat{\ell}_{2}(t) \\
\widehat{n}(t) & =\check{z}_{1}(t) \hat{x}_{1}(t) \\
\widehat{d}(t) & =\left(k_{10}+\check{z}_{1}(t)\right)\left(k_{11}+\hat{x}_{1}(t)\right)
\end{aligned}
$$

The derived functions $\dot{\hat{\ell}}_{3}(t), \dot{\hat{\ell}}_{4}(t)$ of $(40 \mathrm{a})$ and $\dot{\hat{\ell}}_{2}(t), \dot{\hat{z}}_{3}(t)$ of $(40 \mathrm{~h})$ are defined by :

$$
\begin{aligned}
\dot{\hat{\ell}}_{2}(t) & =\frac{\dot{\hat{n}}(t) \hat{d}(t)-\dot{\hat{d}}(t) \hat{n}(t)}{\widehat{d}(t)^{2}} \\
\dot{\hat{n}}(t) & =\dot{\hat{z}}_{1}(t) \hat{x}_{1}(t)+\check{z}_{1}(t) \check{x}_{2}(t) \\
\dot{\hat{d}}(t) & =\dot{\hat{z}}_{1}(t)\left(k_{11}+\widehat{x}_{1}(t)\right)+\check{x}_{2}(t)\left(k_{10}+\check{z}_{1}(t)\right) \\
\dot{\hat{\ell}}_{3}(t) & =u_{22}(t)\left(k_{12}-\widehat{x}_{1}(t)\right)-u_{21}(t) \check{x}_{2}(t) \\
\dot{\hat{\ell}}_{4}(t) & =u_{12}(t) \hat{x}_{1}(t)+u_{11}(t) \check{x}_{2}(t) \\
\dot{\hat{z}}_{1}(t) & =k_{1} \hat{\ell}_{1}(t)+k_{2} \widehat{z}_{3}(t)-k_{3} \hat{\ell}_{2}(t) \widehat{z}_{3}(t) \\
\dot{\hat{z}}_{3}(t) & =k_{9} \widehat{\ell}_{5}(t)-k_{6} \widehat{\ell}_{6}(t)-k_{7} \widehat{z}_{3}(t)+k_{8} \widehat{\ell}_{2}(t) \widehat{z}_{3}(t)
\end{aligned}
$$

The observer in canonical form of regulation of the system(36) is written :

$$
\begin{aligned}
\dot{\hat{x}}_{1}(\tau) & =\breve{x}_{2}(\tau)+h_{2} \Delta y_{1}(\tau) \\
\dot{\check{x}}_{2}(\tau) & =I_{0}(\tau)+h_{1} \Delta y_{1}(\tau)+\widetilde{\Psi}(\tau) \\
\dot{I}_{0}(\tau) & =h_{0} \Delta y_{1}(\tau) \\
\Delta y_{1}(\tau) & =y_{2}(\tau)-\widehat{x}_{1}(\tau)
\end{aligned}
$$

with $y_{2}(\tau)$ (31b) used to form the observation error $\Delta y_{1}(\tau)(14 \mathrm{c})$. 


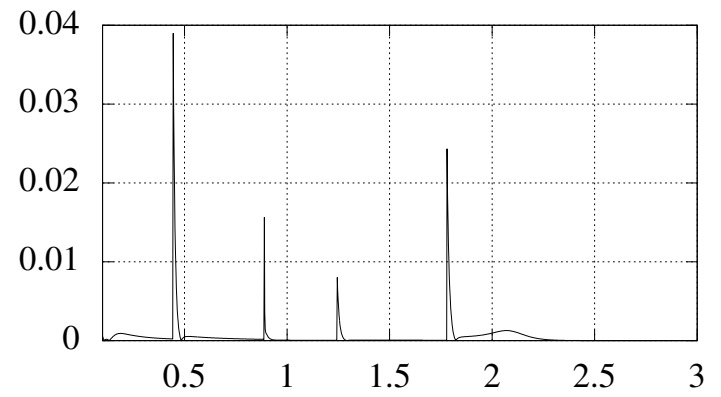

(h) Evolution of $\left\|\Delta \dot{\widetilde{\Psi}}_{1}\left(\tau_{1}\right)\right\| /\left\|\Delta \underline{Z}\left(\tau_{1}\right)\right\|$

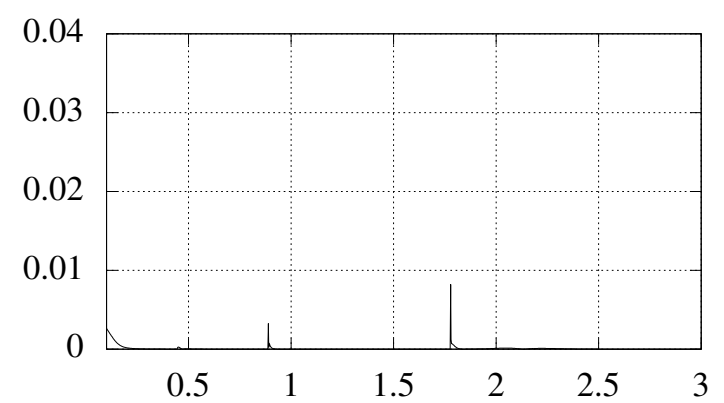

(j) Evolution of $\left\|\Delta \dot{\widetilde{\Psi}}_{2}\left(\tau_{2}\right)\right\| /\left\|\Delta \underline{Z}\left(\tau_{2}\right)\right\|$

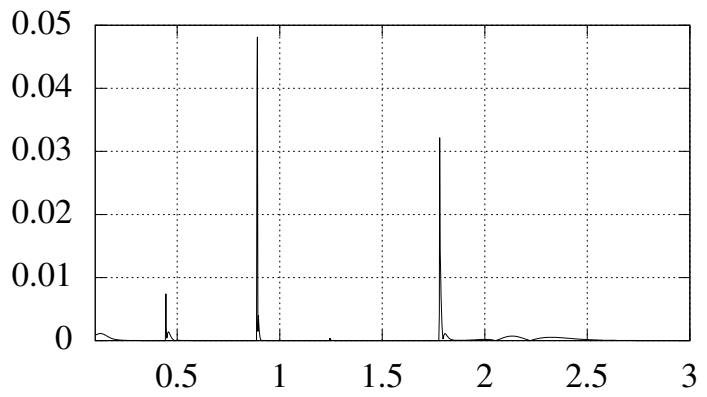

(i) Evolution of $\|\Delta \dot{\widetilde{\Psi}}(\tau)\| /\left\|\Delta \underline{y}_{a}(\tau)\right\|$

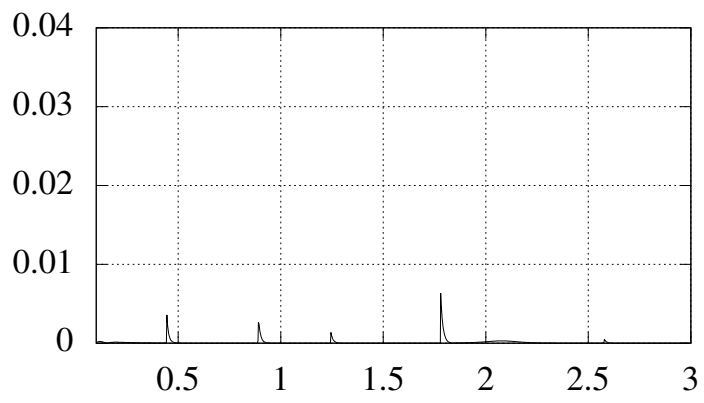

(k) Evolution of $\left\|\Delta \dot{\tilde{\Psi}}_{3}\left(\tau_{3}\right)\right\| /\left\|\Delta \underline{Z}\left(\tau_{3}\right)\right\|$

Figure 4. Search for Lipschitz constants

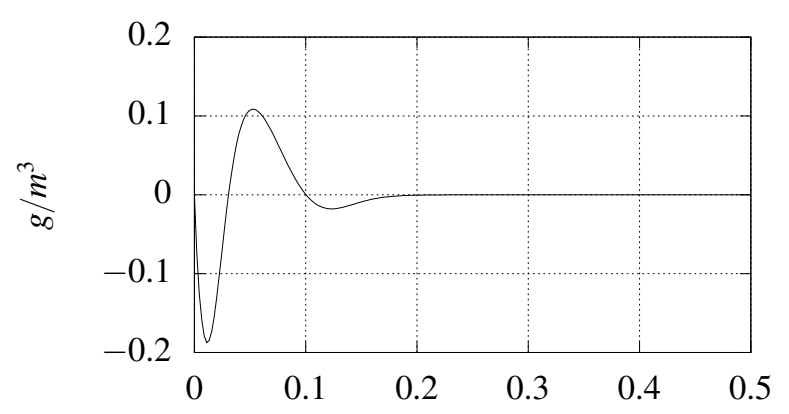

(1) Difference $z_{1}(t)-\check{z}_{1}(t)$ in carbonated substrate

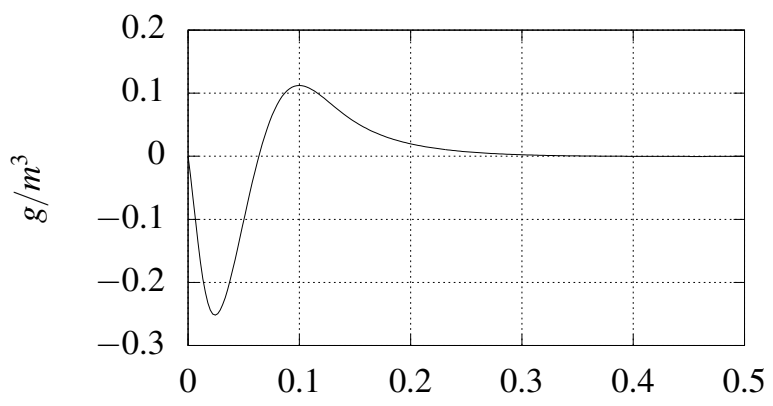

(m) Dissolved oxygen difference $z_{2}(t)-\check{z}_{2}(t)$

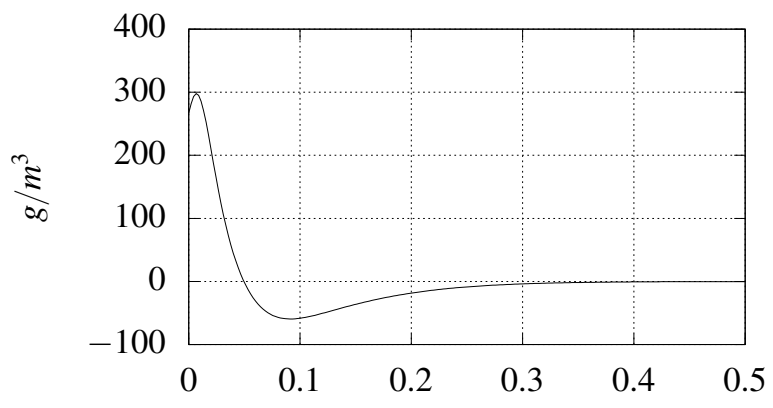

(n) Biomass difference $z_{3}(t)-\check{z}_{3}(t)$

Figure 5. State distances without measurement noise 


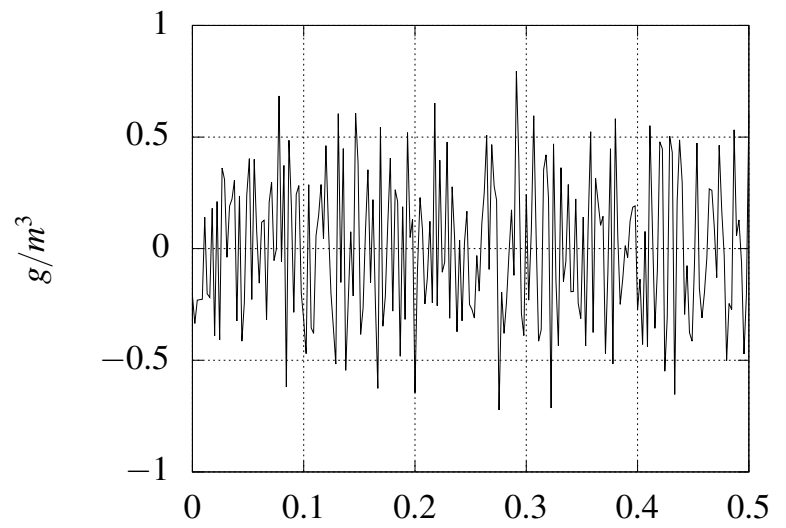

(o) Difference $z_{1}(t)-\check{z}_{1}(t)$ in carbonated substrate

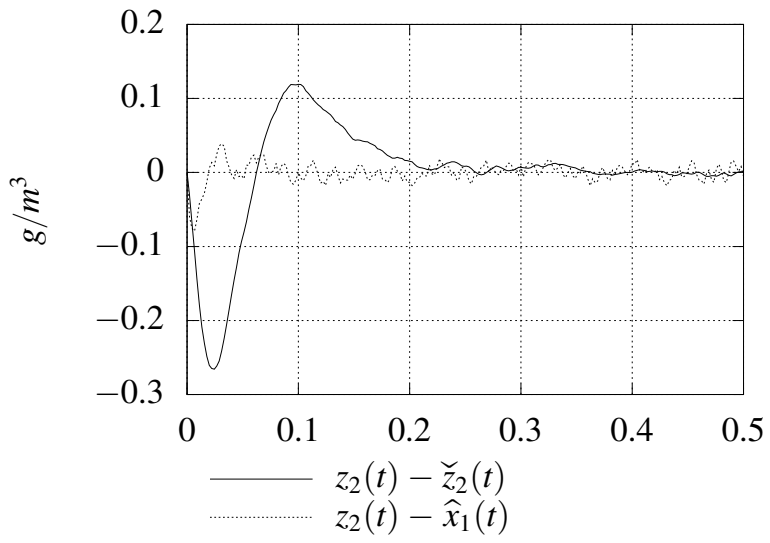

(p) Dissolved oxygen difference $z_{2}(t)-\check{z}_{2}(t)$

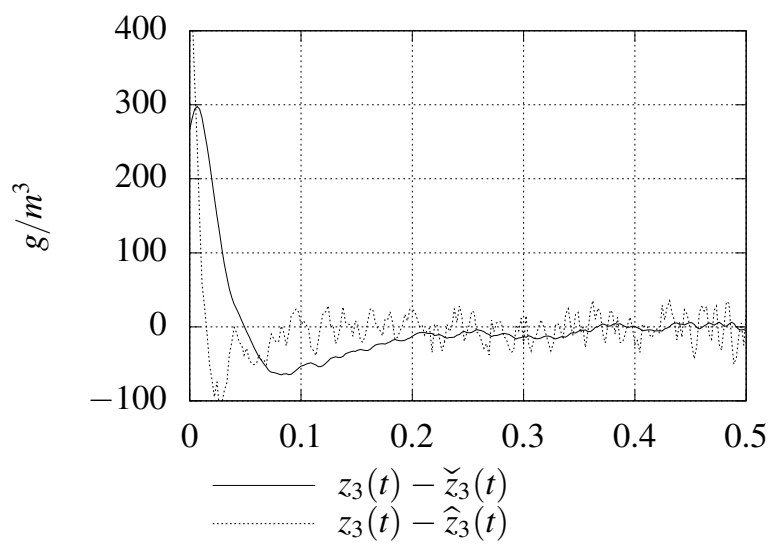

(q) Biomass difference $z_{3}(t)-\breve{z}_{3}(t)$

Figure 6. State distances with measurement noise

The estimate $\hat{z}_{3}(t)$ of $z_{3}(t)$ used in (40h), (41f) and (41g) and also the estimate $\widehat{z}_{2}(t)$ of $z_{2}(t)$ are defined by the following inverted transform functions :

$$
\begin{aligned}
\widehat{z}_{1}(t) & =y_{1}(t) \\
\widehat{z}_{2}(t) & =\widehat{x}_{1}(t) \\
\widehat{z}_{3}(t) & =\frac{k_{4} \widehat{\ell}_{3}(t)-k_{1} \widehat{\ell}_{4}(t)-\breve{x}_{2}(t)}{k_{5} \widehat{\ell}_{2}(t)} \\
\widehat{\hat{z}}(t) & =\left[\begin{array}{lll}
\widehat{z}_{1}(t) & \widehat{z}_{2}(t) & \widehat{z}_{3}(t)
\end{array}\right]
\end{aligned}
$$

\subsection{Observation of the Inverted Transformation System}

The inverted transformation system (43) serves to form the errors (14c) of three first order observers of the same type as those defined in (17), in order to estimate $\widehat{z}(t)$.

The observed outputs and the scaling pulsation choices are given by :

$$
\begin{aligned}
\check{z}(t) & =\left[\begin{array}{lll}
\check{z}_{1}(t) & \check{z}_{2}(t) & \check{z}_{3}(t)
\end{array}\right] \\
\check{s}_{i}\left(\tau_{i}\right) & =\frac{s_{i}\left[\underline{u}_{1}(t), \underline{\underline{z}}(t)\right]}{\omega_{i}} \quad i=1 \ldots 3 \\
\omega_{1} & =\omega_{o} \quad \omega_{2}=\omega_{3}=\omega_{o} / 5
\end{aligned}
$$


the observer of $\widehat{z}_{1}(t)$ is written :

$$
\begin{aligned}
\dot{\check{z}}_{1}\left(\tau_{1}\right) & =I_{1}\left(\tau_{1}\right)+2 \Delta z_{1}\left(\tau_{1}\right)+\check{s}_{1}\left(\tau_{1}\right) \\
\check{s}_{1}\left(\tau_{1}\right) & =\frac{k_{1} \widehat{\ell}_{1}(t)+k_{2} \check{z}_{3}(t)-k_{3} \check{\ell}_{2}(t) \check{z}_{3}(t)}{\omega_{1}} \\
\check{\ell}_{2}(t) & =\frac{\check{z}_{1}(t) \check{z}_{2}(t)}{\left(k_{10}+\check{z}_{1}(t)\right)\left(k_{11}+\check{z}_{2}(t)\right)} \\
\dot{I}_{1}\left(\tau_{1}\right) & =\Delta z_{1}\left(\tau_{1}\right) \\
\Delta z_{1}\left(\tau_{1}\right) & =\widehat{z}_{1}(t)-\check{z}_{1}\left(\tau_{1}\right)
\end{aligned}
$$

That of $\widehat{z}_{2}(t)$ :

$$
\begin{aligned}
\dot{\check{z}}_{2}\left(\tau_{2}\right) & =I_{2}\left(\tau_{2}\right)+2 \Delta z_{2}\left(\tau_{2}\right)+\check{s}_{2}\left(\tau_{2}\right) \\
\check{s}_{2}\left(\tau_{2}\right) & =\frac{k_{4} \check{\ell}_{3}(t)-k_{1} \check{\ell}_{4}(t)-k_{5} \check{\ell}_{2}(t) \check{z}_{3}(t)}{\omega_{2}} \\
\check{\ell}_{3}(t) & =u_{21}(t)\left[k_{12}-\check{z}_{2}(t)\right] \\
\check{\ell}_{4}(t) & =u_{11}(t) \check{z}_{2}(t) \\
\dot{I}_{2}\left(\tau_{2}\right) & =\Delta z_{2}\left(\tau_{2}\right) \\
\Delta z_{2}\left(\tau_{2}\right) & =\widehat{z}_{2}(t)-\check{z}_{2}\left(\tau_{2}\right)
\end{aligned}
$$

that of $\widehat{z}_{3}(t)$ :

$$
\begin{aligned}
\dot{\check{z}}_{3}\left(\tau_{3}\right) & =I_{3}\left(\tau_{3}\right)+2 \Delta z_{3}\left(\tau_{3}\right)+\check{s}_{3}\left(\tau_{3}\right) \\
\check{s}_{3}\left(\tau_{3}\right) & =\left[\begin{array}{c}
k_{9} \hat{\ell}_{5}(t)-k_{6} u_{11}(t) \check{z}_{3}(t) \\
-k_{7} \check{z}_{3}(t)+k_{8} \check{\ell}_{2}(t) \check{z}_{3}(t)
\end{array}\right] / \omega_{3} \\
\dot{I}_{3}\left(\tau_{3}\right) & =\Delta z_{3}\left(\tau_{3}\right) \\
\Delta z_{3}\left(\tau_{3}\right) & =\widehat{z}_{3}(t)-\check{z}_{3}\left(\tau_{3}\right)
\end{aligned}
$$

The observer (45) is there to counteract the effect of measurement noise superimposed on $z_{1}(t)$, which has sometimes a very great impact on the estimates $\widehat{z}_{3}(t)(43 \mathrm{c})$, due to the term $\hat{\ell}_{2}(t)$ in the denominator.

The scaling of (46b) and (47b), parts of (45b) is defined in (44c). This has for effect to strongly reduce the noise on estimates $\check{z}_{2}(t)$ and $\check{z}_{3}(t)$.

We now try to determine the Lipschitz constants that subsequently will allow defining the stability conditions of each observer. We thus start by looking for $L$ in (36) using the same calculation method as that explained in ((Schwaller, Ensminger, Dresp-Langley, \& Ragot, 2016), section 3.1).

With (31)-(34) and (38c) we get $x_{2}(t)$ by using $\underline{z}(t), \ell_{2}(t) \ell_{3}(t)$ and $\ell_{4}(t)$. Then it is possible with (36c) and (38b) to calculate $\underline{x}(t)$ and then $\widetilde{\Psi}[x(t), \underline{U}(t)] / \omega_{o}^{2}$.

Using the initial conditions $\underline{z}(0)$ with the same method of calculation, we can determine $\widetilde{\Psi}[\underline{x}(0), \underline{U}(t)] / \omega_{o}^{2}$. We then calculate the state distance $\Delta \bar{y}(\tau)$. Numerical derivation of $\widetilde{\Psi}[\underline{x}(t), \underline{U}(t)] / \omega_{o}^{2}-\widetilde{\Psi}[\underline{x}(0), \underline{U}(t)] / \omega_{o}^{2}$ permits determination of the augmented vector $\underline{y}_{a}(\bar{\tau})$ of observation error and to obtain a Lipschitz constant adapted to the observer (42). Figure 4 (b) page 13 illustrates this procedure and allows choosing a constant $L=0.15$. The abscissa is represented only for the first three hours of recording, the region where convergence of observers is expected. using the same stability conditions explained in ((Schwaller, Ensminger, Dresp-Langley, \& Ragot, 2016), section 2.3 and 2.4), we fix parameter $\phi_{3}=2, \phi_{2}=2, \phi_{1}=4$. We choose $\lambda=1 / 8, \sigma=1$ and obtain the limiting conditions to respect to synthesise the gains $h_{i}$ :

$$
h_{0} \geqslant 0.273 \quad h_{1} \geqslant 0.512 \quad h_{2} \geqslant 1.3175
$$

Using $v=1$ and $n=2$, we get :

$$
\underline{h}_{a}=\left[\begin{array}{lll}
1 & 3 & 3
\end{array}\right]
$$

which respects the conditions (48). 
We now try to determine constants $L_{1}, L_{2}, L_{3}$ of inverted transformation observer functions. We use a similar procedure to estimate $\Delta \underline{Z}\left(\tau_{i}\right), \Delta \dot{\tilde{\Psi}}_{i}\left(\tau_{i}\right)$ and their respective modules. Figures 4(a),(c) and (d) allows the choice of Lipschitz constants $L_{1}=0.1, L_{2}=L_{3}=0.02(22)$.

If we fix $\phi_{i 1}=\phi_{i 2}=2, \sigma_{i}=1, n=3, \lambda_{i}=1 / 8$ for $i=1 \ldots 3$ the stability conditions (23) are respected, and the three observers of $\underline{\hat{g}}(\tau)$ will be stable and properly damped.

\subsection{Simulations and Results Obtained}

The aim of the simulation is to observe the overall stabilisation of observers to an initial difference in biomass concentration.

The initial conditions of (39) are fixed at

$$
I_{0}(0)=0, \breve{x}_{2}(0)=-0.164, \widehat{x}_{1}(0)=z_{2}(0)=3
$$

Those of $(45)$ at $\check{z}_{1}(0)=z_{1}(0)=4.1$, those of $(46)$ at $\check{z}_{2}(0)=3$, those of $(47)$ at $\check{z}_{3}(0)=600$.

In figures 5(a)- 5(c) the exponential convergent reduction of the state distances $z_{i}(t)-\breve{z}_{i}(t)$ are visualised for zero measured noise on the outputs $y_{1}(t)$ and $y_{2}(t)$. The same test is performed by adding two bandwidth limited white noise to outputs $y_{1}(t)$ and $y_{2}(t)$. These uncorrelated noises have an amplitude of $1 \%$ on each of the variables. Figure 4(h) permits verification that the dynamics of convergence of $\check{z}_{3}(t)$ is conserved. The normative pulse $\omega_{o}$ chosen for (39) and (45) allow reduction of residual noise by about $10 \%$ compared with the measured variables and to contain that still present in $\widehat{z}_{3}(t)$. This setting permits however to have a rate of convergence of $\check{z}_{1}(t)$ and $\hat{x}_{1}(t)$ of the same order as the abrupt variations that are seen in $\left.z_{1} t\right)$ and $z_{2}(t)$.

In figures 6(a)-(c) page 14 the smoothing effect on the estimates of observer (46) and (47) is illustrated : division by 5 on the noise on $y_{2}(t)$ for $\check{z}_{2}(t)$ and fluctuations of $2 \%$ on superimposed noise compared with the full scale for $\check{z}_{3}(t)$.

\section{Conclusions and Perspectives}

Observation in canonical form of regulation that is proposed in (Schwaller, Ensminger, Dresp-Langley, \& Ragot, 2016) did not take into account the effect of measurement noise on the inverted transformation, which allowed passing from the observation of transformed systems to the non-transformed state space. Certain non-linear functions, because of their nature, can greatly amplify the effect of extraneous perturbations on the final estimations. Observers of inverted transformation functions limit this type of effect. The time scale of each observer affects the stability conditions of each observer, via the value of the Lipschitz constant. This also greatly influences the existing noise on the estimated variables. By reducing the pulse $\omega_{i}$ of the observers (17), the Lipschitz constant $L_{i}$ is reduced, and similarly the magnitude of remaining noise on estimates $\check{z}_{i}(t)$ and one increases the convergence time. Setting the rate of convergence of each observer can be done independently.

Observer stability and synthesising observer gains employ demonstrations published in the previous study.

The proposed technique can be applied to other observers (Gauthier, Hammouri, \& Othman, 1992) or to different high gain observers. Observation of inverted transformation functions opens the route to identification on line of parameters of $n$ equation of the state of vector $\underline{s}(t)(1 \mathrm{c})$. In fact, it is possible to consider using the $n$ functional distances between $\dot{\widehat{z}}_{i}(t)$ and $s_{i}\left[\underline{u}_{1}(t), \underline{z}(t)\right]$ to identify parameters of the $n$ functions $s_{i}\left[\underline{u}_{1}(t), \underline{z}(t)\right](1 \mathrm{c})$. This could provide a means of dealing with parametric uncertainty in state equations of the system (1), as well as external perturbations, which are already compensated by the integral component of the observer $(14 \mathrm{e})$.

Finally, by slightly modifying the filter (8)-(10), it can be envisaged to extend the proposed method to multivariable MIMO systems with multiple outputs.

\section{References}

Back, J., \& Seo J. (2008). Constructive algorithm for system immersion into non-linear observer form, International Journal of Control, 81(2), 317-331. https://doi.org/10.1080/00207170701559199

Bestle, D., \& Zeitz, M. (1983). Canonical form observer design for non-linear time-variable systems, International Journal of Control, 38(2), 419-431. https://doi.org/10.1080/00207170701559199

Bezzaoucha, S., Marx, B., Maquin, D., \& Ragot, J. (17-19 June 2013). State and parameter estimation for timevarying systems: a Takagi-Sugeno approach. In American Control Conference (ACC), Washington DC, 1050-1055. https://doi.org/10.3182/20130204-3-FR-2033.00106

Bodizs, L., Srinivasan, B., \& Bonvin, D. (March 2011). On the design of integral observers for unbiased output estimation in the presence of uncertainty, Journal of Process Control, 21(3), 379-390.

https://doi.org/10.1016/j.jprocont.2010.11.015 
Boker, A., \& Khalil, H. (December 2013). Nonlinear observers comprising high-gain observers and extended Kalman filters, Automatica, 49(12), 3583-3590. https://doi.org/10.1016/j.jprocont.2010.11.015

Ciccarella, G., Mora, M.D., \& Germani, A. (1993). A Luenberger-like observer for nonlinear systems, International Journal of Control, 57(3), 537-556. https://doi.org/10.1080/00207179308934406

Efimov, D., \& Fridman, L. (March 2011). Global sliding-mode observer with adjusted gains for locally Lipschitz systems, Automatica, 47(3), 565-570. https://doi.org/10.1016/j.automatica.2010.12.003

Farza, M., Bouraoui, I., Ménard, T., Abdennour, R.B., \& MSaad, M. (November 2014). Adaptive observers for a class of uniformly observable systems with nonlinear parametrization and sampled outputs, Automatica, 50(11), 2951-2960. https://doi.org/10.1016/j.automatica.2014.10.032

Fliess, M. (September 1990). Generalized controller canonical forms for linear and nonlinear dynamics, IEEE Transactions on Automatic Control, 35(9), 994-1001. https://doi.org/10.1109/9.58527

Gauthier, J., Hammouri, H., \& Othman, S. (June 1992). A simple observer for nonlinear systems. applications to bioreactors, IEEE Transactions on Automatic Control, 37(6), 875-880. https://doi.org/10.1109/9.256352

Guerra, T.M., Estrada-Manzo, V., \& Lendek, Z. (February 2015). Observer design for Takagi Sugeno descriptor models: an lmi approach, Automatica, 52: 154-159. https://doi.org/10.1109/AQTR.2014.6857823

Ibrir, S. (October 2009). Adaptive observers for time-delay nonlinear systems in triangular form, Automatica, 45(10), 2392 2399. https://doi.org/10.1016/j.automatica.2009.06.027

Khalifa, T., \& Mabrouk, M. (January 2015). On observer for a class of uncertain nonlinear systems, Nonlinear Dynamics, 79(1), 359-368. https://doi.org/10.1007/s11071-014-1669-6

Krener, A., \& Isidori, A. (1983). Linearization by output injection and nonlinear observers, Systems $\mathcal{E}$ Control Letters, 3(1), 47-52. https://doi.org/10.1007/s11071-014-1669-6

Luenberger, D. (1966). Observers for multivariable systems, IEEE Transactions on Automatic Control, AC-11(2), 190 197, https://doi.org/10.1007/s11071-014-1669-6

Martínez-Guerra, R., Mata-Machuca, J., Aguilar-López, R., \& Rodríguez-Bollain, A. (2011). Applications of Chaos and Nonlinear Dynamics in Engineering, 1. Springer-Verlag Berlin Heidelberg. ISBN 978-3-642-21921-4. https://doi.org/10.1007/978-3-642-21922-1

Mazenc, F., \& Dinh, T. (October 2014). Construction of interval observers for continuous-time systems with discrete measurements, Automatica, 50(10), 2555-2560. https://doi.org/10.1016/j.automatica.2014.08.008

Menini, L., \& Tornambè, A. (2011). Design of state detectors for nonlinear systems using symmetries and semi-invariants, Systems and Control Letters, 60(2), 128-137. https://doi.org/10.1016/j.sysconle.2010.11.004

Mobki, H., Sadeghia, M., \& Rezazadehb, G. (April 2015). Design of direct exponential observers for fault detection of nonlinear mems tunable capacitor, IJE Transactions A : Basics, 28(4), 634-641. https://doi.org/10.1016/j.sysconle.2010.11.004

Morales, A., \& Ramirez, J. (May 2002). A pi observer for a class of nonlinear oscillators, Physics Letters A, 297(34), 205-209. https://doi.org/10.1016/S0375-9601(02)00191-3

Nagy-Kiss, A., Marx, B., Mourot, G., Schutz, G., \& Ragot, J. (15-17 Dec. 2 2010). State estimation of two-time scale multiple models with unmeasurable premise variables. application to biological reactors. In 49th IEEE Conference on Decision and Control (CDC), 5689-5694. Atlanta, GA, USA. https://doi.org/10.1109/CDC.2010.5718035

Rauh, A., Butt, S., \& Aschemann, H. (2013). Nonlinear state observers and extended Kalman filters for battery systems, International Journal of Applied Mathematics and Computer Science, 23(3), 539-556. https://doi.org/10.2478/amcs2013-0041

Röbenack, K., \& Lynch, A. (May 2004). An efficient method for observer design with approximately linear error dynamics, International Journal of Control, 77(7), 607-612. https://doi.org/10.1080/00207170410001682515

Röbenack, K., \& Lynch, A. (September 2006). Observer design using a partial nonlinear observer canonical form, International Journal of Applied Mathematics and Computer Science, 16(3), 333-343.

Schwaller, B., Ensminger, D., Dresp-Langley, B., \& Ragot, J. (June 2013). State estimation for a class of non-linear systems, International Journal of Applied Mathematics and Computer Science, 23(2), 383-394.

https://doi.org/10.2478/amcs-2013-0029 
Schwaller, B., Ensminger, D., Dresp-Langley, B., \& Ragot, J. (September 2016). State estimation for miso non-linear systems in generalized canonical form of regulation, International Journal of Applied Mathematics and Computer Science, 26(3), 569-583.

https://doi.org/10.1515/amcs-2016-0040

Söffker, D., Yu, T., \& Müller, P. (1995). State estimation of dynamical systems with nonlinearities by using proportionalintegral observers, International Journal of Systems Science, 26(9), 1571-1582. https://doi.org/10.1080/00207729508929120

Thabet, R., Raïssi, T., Combastel, C., Efimov, D., \& Zolghadri, A. (October 2014). An effective method to interval observer design for time-varying systems, Automatica, 50(10), 2677-2684. https://doi.org/10.1016/j.automatica.2014.08.035

Tornambè, A. (1992). High-gain observers for non-linear systems, International Journal of Systems Science, 23(9), 14751489. https://doi.org/10.1080/00207729208949400

Tyukina, I., Steurb, E., Nijmeijerc, H., \& van Leeuwenb, C. (August 2013). Adaptive observers \& parameter estimation for a class of systems nonlinear in the parameters, Automatica, 49(8), 2409-2423. https://doi.org/10.1016/j.automatica.2013.05.008

Zheng, G., Boutat, D., \& Barbot, J. (January 2009). Multi-output dependent observability normal form, Nonlinear Analysis : Theory, Methods E Applications, 70(1), 404-418. https://doi.org/10.1016/j.na.2007.12.012

\section{Copyrights}

Copyright for this article is retained by the author(s), with first publication rights granted to the journal.

This is an open-access article distributed under the terms and conditions of the Creative Commons Attribution license (http://creativecommons.org/licenses/by/4.0/). 